\title{
The role of the central chemoreceptor in causing periodic breathing
}

\author{
A. C. FOWLER \\ Mathematical Institute, University of Oxford, 24-29 St. Giles', Oxford OXI 3LB, \\ $U K$ \\ AND \\ G. P. Kalamangalam \\ Department of Neurology, St. James's University Hospital, Leeds LSS TTF, UK
}

[Received 7 April 1999]

\begin{abstract}
In a previous publication (Fowler et aL, 1993), we reduced the classical cardiorespiratory control model of (Grodins et al, 1967) to a much simpler form, which we then used to study the phenomenon of periodic breathing. In particular, cardiac output was assumed constant, and a single (constant) delay representing arterial blood transport time between lung and brain was included in the model. In this paper we extend this earlier work, both by allowing for the variability in transport delays, due to the dependence of cardiac output on blood gas concentrations, and also by including further delays in the system. In addition, we extensively discuss the physiological implications of parameter variations in the model; several novel mechanisms for periodic breathing in clinical situations are proposed. The results are discussed in the light of recent observational studies.
\end{abstract}

Keywords: Periodic breathing; Cheyne-Stokes respiration; heart-rate variability; differential-delay equations.

\section{Introduction}

Periodic breathing (PB) is a slow waxing and waning of the amplitude of respiration, which typically cycles over a time scale between $30 \mathrm{~s}$ and $3 \mathrm{~min}$. It is a naturally occurring phenomenon, particularly in adults at high altitude, and is associated with corresponding fluctuations in heart rate and blood pressure through the interactions of these quantities in the cardio-respiratory system.

In its more extreme forms, periodic breathing is of clinical concem. In particular, Cheyne-Stokes respiration (CSR) is a severe form of periodic breathing in which the waxing and waning respiratory cycles are interrupted by periods of apnoea, when breathing ceases altogether, and CSR is routinely found, for example, to occur in patients with heart failure (Mortara et al., 1997; Quaranta et al., 1997). This suggests that the failing heart provides one mechanism whereby such oscillations can be caused. This is borne out by the study of heart-rate variability (HRV). Physiologists recognize three major peaks in the Fourier spectrum of 24-h HRV data sets: a high frequency $(>0.2 \mathrm{~Hz}$ ) band attributable to the basic respiratory rhythm (often known as sinus anthythmia), a low frequency ( 0.04 $0.2 \mathrm{~Hz}$ ) band attributed to interaction with the baroreceptor control mechanisms, and a very low frequency $(<0.04 \mathrm{~Hz}$ ) band, which is thought to arise from thermoregulation 
and respiratory chemical feedback mechanisms (Kitney \& Rompelman, 1987). Several authors (Goldberger et al, 1984; Kaplan \& Talajic, 1991; Mortara et al, 1997) document an increase in very low frequency power in patients with chronic left heart failure, and relate this to the occurrence of periodic breathing.

Mathematical models of periodic breathing have been developed by many authors (Mackey \& Glass, 1977; Khoo et al, 1982; Carley \& Shannon, 1988; Longobardo et ah. 1989; El Hefnawy et al., 1990). Most simply stated, the onset of periodic breathing can be understood by a simple delay-differential equation (DDE) model which represents the respiratory control exerted by $\mathrm{CO}_{2}$-sensitive chemoreceptors; the control is delayed by the transport time for the blood to travel from the lungs to the chemoreceptor. It is well known that increasing delays lead to oscillatory instabilities in DDEs, and in the present case, increased delay is associated with the decreased cardiac output of heart failure. It follows that any realistic model of the respiratory system ought to include a description of (a varying) cardiac output as a constituent. The latter is primarily controlled via arterial baroreceptors that respond to variations in blood pressure; however, significant fluctuations in blood gas tensions also play a role. This coupling between respiratory chemoreflex and cardiac output has the mathematical effect of causing the delays due to blood transport to be 'state-dependent'. In this paper, we will include a study of the extra complication afforded by such variable delays.

Our purpose is to build and analyse a model for chemoreflex respiratory control that allows for the dependence of cardiac output on blood gas tensions. We base our study on previous work (Fowler et al., 1993), which showed how the classical model of Grodins et al. (1967) could be effectively condensed to a much simpler (though still very complex) form. In its most extreme simplification, this model can be reduced to a single delay-recruitment model, which exhibits oscillatory instability as the delay parameter is increased. In the present paper, we will extend these results to include the effect of variable cardiac output, which leads to state-dependent delays, and we will also allow for separate delays describing arterial transport time between lungs and brain, lungs and tissues, and venous transport time from tissues and brain to lungs. Our aim is to show how the presence of variable, multiple delays affect the nature and onset of periodic breathing, and also how the cycling of blood flow is naturally associated with it.

The remainder of the paper is organized as follows. In Section 2, we rehearse the form of the Grodins model derived by Fowler et al (1993), and recall how the one-delay model was derived; here we pose the more accurate model of a pair of coupled delayrecruitment equations for tissue $\mathrm{CO}_{2}$ and brain $\mathrm{CO}_{2}$ concentration-but delayed by the transit times to tissue and brain, respectively. In turn, the arterial $\mathrm{CO}_{2}$ rapidly relaxes towards an equilibrium determined in terms of (delayed) tissue and brain $\mathrm{CO}_{2}$, and which depends also on current brain $\mathrm{CO}_{2}$ through the ventilation response curve.

The model is analysed in Section 3, and the results compared with those of the simpler model, and conclusions and comparisons with clinical observations are made in Section 4.

\section{A reduced Grodins model}

The model of Grodins et al. (1967) is a compartment model which relates the dependence of ventilation $\dot{V}$ on $\mathrm{CO}_{2}$ and $\mathrm{O}_{2}$ levels in the blood. Chemical control of ventilation is effected through the action of central (in the brainstem) and peripheral (in the carotid artery 
in the neck) chemoreceptors. Ventilation does not change significantly with $\mathrm{O}_{2}$ until the partial pressure drops below about $70 \mathrm{~mm} \mathrm{Hg}$. Since normal levels are $100 \mathrm{~mm} \mathrm{Hg}$, this suggests that ventilation is primarily controlled by $\mathrm{CO}_{2}$ levels. In fact, Fowler et al. (1993) showed that $\mathrm{O}_{2}$ concentrations would typically relax rapidly towards equilibrium, which suggests that in normal circumstances, the effects of $\mathrm{O}_{2}$ variation can be ignored, and we make this assumption here also.

There are three principal compartments in the model: lungs, tissues, brain. These are connected by the blood flow in arteries (from lungs to tissues and brain) and by the return venous flow. In the model one visualizes the arterial blood flow sweeping past the lungs, where gas exchange occurs, whereas in fact this occurs via the pulmonary circulation from the right heart to the lungs to the left heart (and thence to the arteries). The blood flow to the brain and tissues is in parallel, and the transport time between the compartments leads to four natural delay times, denoted $\tau_{\mathrm{ab}}, \tau_{\mathrm{at}}, \tau_{\mathrm{vb}}$ and $\tau_{\mathrm{v}}$, which are, respectively, the arterial transport times to brain and tissues, and the subsequent venous transport times back to the lungs (via the right heart).

As explained at the end of Section 1, we assume that $\mathrm{CO}_{2}$ variations are the only ones of significance. Let $c_{\mathrm{b}}, c_{\mathrm{t}}, c_{1}$ denote the concentrations of $\mathrm{CO}_{2}$ at brain, tissues and lungs (i.e. in the arterial blood at the lungs). As in our previous work, we suppose that these variables and the others have been non-dimensionalized using typical resting values, which are denoted by square brackets (time is initially arbitrarily scaled with $[t]=1 \mathrm{~min}$ ): thus $\left[c_{1}\right]$ denotes a typical value of the arterial blood exiting the lung, etc.

Before we begin, we indicate typographical or other errors in Fowler et al. (1993) in Table 1 . The change from $p_{27}$ to $p_{26}$ affects (numerically) the discussion below. Fowler $e t$ al. (1993) showed that it was reasonable to suppose that blood flow $Q$ and brain blood flow $Q_{b}$ equilibrated rapidly to values determined by the arterial $\mathrm{CO}_{2}$ concentrations $c_{1}$, thus

$$
Q \approx Q\left(c_{1}\right), \quad Q_{\mathrm{b}} \approx Q_{\mathrm{b}}\left(c_{1}\right),
$$

while the ventilation rate is taken to depend solely on brain $\mathrm{CO}_{2}$, thus

$$
\dot{V} \approx \dot{V}\left(c_{b}\right)
$$

since the arterial $\mathrm{O}_{2}$ concentration remains at equilibrium at a value above where $\dot{V}$ depends on it.

The arterial $\mathrm{CO}_{2}$ concentration $c_{l}$ relaxes rapidly to an equilibrium given by equation (16) of Fowler et al:

$$
c_{l}=\frac{p_{0} \dot{V}+\Lambda_{1}\left[p_{13} Q_{\mathrm{b}} c_{\mathrm{b}}\left(t-\tau_{\mathrm{vb}}\right)+\left(p_{24} Q-p_{13} Q_{\mathrm{b}}\right) c_{t}\left(t-\tau_{\mathrm{v}}\right)\right]}{p_{1} \dot{V}+\Lambda_{2} Q} .
$$

The provenance of this equation is easy to understand. It begins life as the balance law

$$
\dot{c}=\left(p_{0}-p_{1} c_{1}\right) \dot{V}+Q\left[\Lambda_{1} c_{v}-\Lambda_{2} c_{2}\right] .
$$

The terms represent, respectively, the acquisition of $\mathrm{CO}_{2}$ by the inspiring lungs $\left(p_{0} \dot{V}\right)$ : normally $p_{0}=0$ unless $\mathrm{CO}_{2}$ is supplied in the atmosphere; the loss term $p_{1} c_{1} \dot{V}$ represents the atmospheric flushing of $\mathrm{CO}_{2}$ by the expiring lungs; the terms proportional to $Q$ 
TABLE 1

Corrections to Fowler et al. (1993)

\begin{tabular}{lcc}
\hline Equation (43) & $\Lambda_{6} Q\left(x_{\mathrm{at}} \rightarrow Q\left(\Lambda_{6} x_{\mathrm{at}}\right.\right.$ \\
Equation (55) & $p_{32} \rightarrow p_{38}$ \\
Equation (87) & $\Lambda_{22} \rightarrow-\Lambda_{22}$ \\
Equation (103) & $p_{27} \rightarrow p_{26}$ \\
Definition & $p_{32}=\frac{\left[Q_{\mathrm{b}}\right]\left[x_{\mathrm{vb}}\right]}{[Q]\left[x_{\mathrm{v}}\right]} \sim 0.11$ \\
Definition & $p_{38}=\frac{\left[c_{\mathrm{vb}}\right]}{\alpha_{8}\left[C_{\mathrm{b}}\right]}=\frac{\left[c_{\mathrm{t}}\right]}{\alpha_{8}\left[C_{\mathrm{t}}\right]} \sim 1.36$ \\
Definition & $p_{45}=\frac{\left[Q_{\mathrm{c}}\right]}{[U]} \sim 0.29$ \\
Definition & $\varepsilon_{11}=\frac{k \sigma_{1} \beta_{1}\left[c_{1}\right]}{\left[c_{\mathrm{a}}\right]} \sim 0.046$. \\
\hline
\end{tabular}

represent the incoming venous $\mathrm{CO}_{2}\left(Q \Lambda_{1} c_{\mathrm{v}}\right)$ and the outgoing arterial $\mathrm{CO}_{2}\left(Q \Lambda_{2} c_{\mathrm{a}}\right)$. Here we make a distinction between arterial $\mathrm{CO}_{2}\left(c_{\mathrm{a}}\right)$ and $\mathrm{CO}_{2}$ fraction in the lung $\left(c_{1}\right)$, and these are related by a complicated sequence of algebraic buffering relationships; in essence, however, we can take $c_{a} \approx c_{1}$ with little significant error. Equation (2.3) follows from assuming that (2.4) reverts rapidly to equilibrium (because the parameters $\Lambda_{i}$ are large), and by relating the venous $\mathrm{CO}_{2}$ concentration to the incoming flows from tissues and brain.

It should be noted that, since $c_{v}$ depends on $c_{b}$ and $c_{t}$ at earlier times, the assumption of equilibrium $c_{1}$ in (2.3) relies on the absence of rapid (chaotic) oscillations in $c_{1}$. Although such rapid oscillations are known to occur in delay equations (Farmer, 1982), we consider them unlikely here, since in fact $c_{\mathrm{t}}$ and $c_{\mathrm{b}}$ are relatively slowly varying, which suggests that (2.3) should be accurate.

The equations for the brain and tissue $\mathrm{CO}_{2}$ concentrations are also simple conservation statements:

$$
\begin{aligned}
& \dot{c}_{\mathrm{t}}=p_{8} Q\left[c_{1}\left(t-\tau_{\mathrm{at}}\right)-c_{\mathrm{t}}\right], \\
& \dot{c}_{\mathrm{b}}=p_{6} Q_{\mathrm{b}}\left[c_{1}\left(t-\tau_{\mathrm{ab}}\right)-c_{\mathrm{b}}\right]
\end{aligned}
$$

these equations are self-explanatory. Finally the transport delays are given by a sequence of functional relationships:

$$
\begin{aligned}
& \varepsilon_{24}=\int_{t-\tau_{\mathrm{b}}}^{t-\delta_{2}} Q_{\mathrm{b}} \mathrm{d} t, \quad \varepsilon_{25}=\int_{t-\delta_{2}}^{t} Q \mathrm{~d} t ; \\
& p_{56}=\int_{t-\tau_{\mathrm{n}}}^{t-\delta_{3}}\left(Q-p_{26} Q_{\mathrm{b}}\right) \mathrm{d} t, \quad \varepsilon_{25}=\int_{t-\delta_{3}}^{t} Q \mathrm{~d} t ; \\
& p_{55}=\int_{t-\tau_{\mathrm{bb}}}^{t-\delta_{0}} Q \mathrm{~d} t, \quad \varepsilon_{23}=\int_{t-\delta_{0}}^{t} Q_{\mathrm{b}} \mathrm{d} t ; \\
& p_{55}=\int_{t-\tau_{\mathrm{tat}}}^{t-\delta_{1}} Q \mathrm{~d} t, \quad p_{13}=\int_{t-\delta_{1}}^{t}\left(Q-p_{26} Q_{\mathrm{b}}\right) \mathrm{d} t .
\end{aligned}
$$


These (dimensionless) relationships define the delays $\tau_{v b r} \tau_{v t}, \tau_{a b}, \tau_{a t}$ respectively. The parameters are generally of order $p_{i} \sim \mathrm{O}(1), \varepsilon_{j} \ll 1$ (and $\Lambda_{k} \gg 1$ ); the $\delta_{i} \mathrm{~S}$ are intermediary variables. Estimates of parameters in (2.6)-(2.9) are

$$
\begin{aligned}
& \varepsilon_{24} \sim 0.08, \quad \varepsilon_{25} \sim 0.031, \quad p_{56} \sim 0.49, \quad p_{55} \sim 0.18, \\
& \varepsilon_{23} \sim 0.02, \quad p_{13} \sim 0.12, \quad p_{26} \sim 0.13 .
\end{aligned}
$$

On the basis that $\varepsilon_{i} \ll 1$, and taking also $p_{13} \sim 0.12$ as small, we can approximate the delays well enough as

$$
\begin{aligned}
& \tau_{\mathrm{vb}} \approx 0, \\
& p_{56} \approx \int_{t-\tau_{\mathrm{v}}}^{t}\left(Q-p_{26} Q_{\mathrm{b}}\right) \mathrm{d} t, \\
& p_{55} \approx \int_{t-\tau_{\mathrm{sb}}}^{t} Q \mathrm{~d} t, \\
& p_{55} \approx \int_{t-\tau_{\mathrm{ut}}}^{t} Q \mathrm{~d} t .
\end{aligned}
$$

To make a direct comparison with our previous work, we choose a suitable form for the ventilation controller. The dimensional ventilation function is really characterized by two quantities, the typical resting value of $51 \mathrm{~min}^{-1}$ and the gain, which may be represented by the dimensionless order of magnitude $\mathrm{d} \dot{V} / \mathrm{d} c_{\mathrm{b}}$. In our previous paper (Fowler et al., 1993), we followed Mackey \& Glass (1977) in choosing, rather arbitrarily,

$$
\dot{V}=\frac{\phi c_{\mathrm{b}}^{10}}{1+c_{\mathrm{b}}^{10}}
$$

Kalamangalam (1995) chose a Mackey-Glass type function

$$
\dot{V}=\frac{\phi\left(c_{\mathrm{b}}-\psi\right)^{\xi}}{1+\left(c_{\mathrm{b}}-\psi\right)^{\xi}},
$$

and showed that a good fit to the Grodins controller was afforded by values $\phi=50, \xi=10$, and $\psi=0.35$. Here we dispense altogether with this sigmoidal type of function, but seek a function which satisfies $V=1$ when $c_{b}=1$, and such that $\mathrm{dV} / \mathrm{d} c_{\mathrm{b}} \sim \phi$ for $c_{\mathrm{b}}>1$. A typical value of $\phi$ is $30-40$ (see, for example, Petersen \& Cunningham, 1988), and the response curve is essentially linear for arterial $\mathrm{CO}_{2}$ pressures greater than the normal value of $40 \mathrm{~mm} \mathrm{Hg}$. Given that $\phi \gg 1$, a suitable choice is then

$$
\dot{V}=\phi v\left(c_{\mathrm{b}}\right)
$$

where

$$
v\left(c_{b}\right)=\frac{\ln \left[1+\exp \left\{\phi\left(c_{b}-1\right) \ln 2\right\}\right]}{\phi \ln 2} .
$$

Next we define the parameters

$$
\alpha=\frac{p_{0} \phi}{\Lambda_{2}}, \quad \beta=\frac{\Lambda_{1} p_{13}}{\Lambda_{2}}, \quad \gamma=\frac{\left(p_{24}-p_{13}\right) \Lambda_{1}}{\Lambda_{2}}, \quad \delta=\frac{p_{1} \phi}{\Lambda_{2}} .
$$


Typical values of these parameters inferred from Fowler et al. (1993) are $\alpha \approx 0, \beta \approx 0 \cdot 12$, $\gamma \approx 0.8$, and if $\phi=90$ for the function given by $(2.15)$, then $\delta \approx 6$. Note that $\beta+\gamma \approx 1$, and in fact it is convenient to choose the scales (and thus $\gamma$ ) so that $c_{\mathrm{b}}=1$ in the steady state when $\alpha=0$. Now in terms of $\alpha, \beta, \gamma$ and $\delta$, the lung $\mathrm{CO}_{2}$ concentration (2.3) is

$$
c=\frac{\alpha v\left(c_{\mathrm{b}}\right)+\beta Q_{\mathrm{b}}\left(c_{1}\right) c_{\mathrm{b}}+\left\{(\gamma+\beta) Q\left(c_{\mathrm{l}}\right)-\beta Q_{\mathrm{b}}\left(c_{1}\right)\right\} c_{\mathrm{t}}\left(t-\tau_{\mathrm{vt}}\right)}{Q\left(c_{\mathrm{l}}\right)+\delta v\left(c_{\mathrm{b}}\right)},
$$

so that if $c_{\mathrm{l}}=c_{\mathrm{b}}=c_{\mathrm{t}}=1$ is the steady-state solution, then we must have

$$
\gamma=1+\frac{p_{1}}{\Lambda_{2}}-\beta
$$

It should be pointed out that the resulting typical value of $\gamma$, which is, with $p_{1} / \Lambda_{2}=0.067$, $\gamma \approx 0.95$, differs from the natural value of 0.8 because of our simplification of the natural buffering relationships between $\mathrm{CO}_{2}$ in different parts of the body. There is no great harm in making this simplification. The model equations (2.5) for $c_{\mathrm{t}}$ and $c_{\mathrm{b}}$, together with the equilibrium (2.17), the delays being given by (2.11), and with prescribed functions $Q\left(c_{1}\right)$ and $Q_{\mathrm{b}}\left(c_{1}\right)$, form the basic reduced Grodins model, which we analyse in Section 3.

\section{Analysis}

The first cause for concern is that (2.17) gives a prescription for $c$ which is implicitly defined, essentially $c_{1}=F\left(c_{1}\right)$. In solving the system numerically, we need a reliable strategy to compute $c_{1}$. In addition, we need to be sure that the root we compute is unique, or if not, that we select the correct one. The quasi-equilibrium (2.17) is attained through (effectively) the solution of $v \dot{c_{1}}=-c_{1}+F\left(c_{1}\right)$, with $v$ small, and so stable equilibrium requires $F^{\prime}\left(c_{1}\right)<1$; this then implies that a reliable iterative method to solve (2.17) is through the sequence

$$
c_{1}^{(n+1)}=F\left(c_{1}^{(n)}\right),
$$

and this is in fact what we do. Whether $F^{\prime}\left(c_{1}\right)<1$ everywhere (in which case (3.1) converges globally to the unique root of (2.17)) is less clear. Both $Q$ and $Q_{\mathrm{b}}$ are increasing functions of $c_{1}$, so it is possible but not guaranteed. Clearly if $\beta=\gamma=0$, then $F$ is decreasing, and there will be a unique (stable) fixed point of the differential equation; the iteration (3.1) will only converge to this if $\left|F^{\prime}\right|<1$ though, which will be the case if $Q^{\prime}$ is not too large. Also if $\beta$ and $\gamma$ are large then $F^{\prime}$ will be large for $Q^{\prime}, Q_{\mathrm{b}}^{\prime}>0:$ (3.1) will not converge, though then the model would predict indefinitely increasing $c$. In practice, however, for the sorts of values of $\beta$ and $\gamma$ and the functional choices for $Q$ and $Q_{b}$ which are used below, $c_{1}$ does appear to be uniquely determined by (2.17).

\subsection{Cardiac output}

The functions used by Grodins et al. (1967) and Fowler et al (1993) (their Fig. 3(a) and (c)) for $Q$ and $Q_{b}$ motivate the following choices:

$$
\begin{aligned}
Q & =1+p\left(c_{1}-1\right)_{+}, \\
Q_{\mathrm{b}} & =1+\Lambda\left(c_{1}-1\right)_{+},
\end{aligned}
$$


with values

$$
p \sim 2, \quad \Lambda \sim 10.6 .
$$

In practice we use smoothed versions, in order to facilitate the use of Runge-Kutta solvers for the differential equations:

$$
\begin{aligned}
Q & =1+\bar{\delta} p \ln \left[1+\exp \left\{\left(c_{1}-1\right) / \bar{\delta}\right\}\right], \\
Q_{\mathrm{b}} & =1+\bar{\delta} \Lambda \ln \left[1+\exp \left\{\left(c_{1}-1\right) / \bar{\delta}\right\}\right],
\end{aligned}
$$

with $\bar{\delta} \ll 1: \bar{\delta}=0.1$ is satisfactory.

\subsection{The FKK reduction}

Fowler et al (1993) simplified the model further by observing that, with typical values, $p_{8} \approx 0.15, p_{6} \approx 0.67$, then $p_{8} \ll p_{6}$, so $c_{1}$ is relatively slowly varying; $c_{b}$ and thus $c_{1}$ vary more rapidly, and averaging part 1 of $(2.5)_{1}$ then implies that $c_{t} \rightarrow \bar{c}_{1}$, where we suppose the running mean $\bar{c}_{1}$ of $c_{1}$ is a constant. Then (2.17) gives

$$
c_{1}=f\left(c_{b}\right) \text {. }
$$

More strictly, $c_{\mathrm{l}}=g\left(c_{\mathrm{b}}, c_{\mathrm{l}}\right)=g\left(c_{\mathrm{b}}, \overline{c_{1}}\right)$, so that $\overline{c_{1}}=\overline{g\left(c_{b}, \bar{c}_{1}\right)}$, which in principle gives $\bar{c}_{1}$ functionally in terms of $c_{\mathrm{b}}$ : in practice we simply take $c_{\mathrm{t}}$ as prescribed.

We then have

$$
\dot{c}_{\mathrm{b}}=p_{6} Q_{\mathrm{b}}\left[f\left(c_{\mathrm{b}}\right)\right]\left[f\left\{c_{\mathrm{b}}\left(t-\tau_{\mathrm{ab}}\right)\right\}-c_{\mathrm{b}}\right],
$$

which is a delay-recruitment equation for $c_{b}$ with variable delay $\tau_{a b}$, given by

$$
p_{55}=\int_{t-\tau_{\text {sb }}}^{t} Q \mathrm{~d} t .
$$

A remarkable transformation due to Smith (1993) can be used to convert this to a constant-delay equation. Define

$$
\tau(t)=\frac{1}{p_{55}} \int_{0}^{t} Q\left[f\left\{c_{b}(s)\right\}\right] \mathrm{d} s ;
$$

it follows, using (3.7), that

$$
\tau\left(t-\tau_{a b}\right)=\tau(t)-1
$$

Now if we define $C(\tau)$ by

$$
C(\tau(t))=c_{\mathrm{b}}(t),
$$

then we find that (3.6) can be written as the constant delay equation

$$
\varepsilon \frac{\mathrm{d} C}{\mathrm{~d} \tau}=q(C)\left[f\left(C_{1}\right)-C\right]
$$




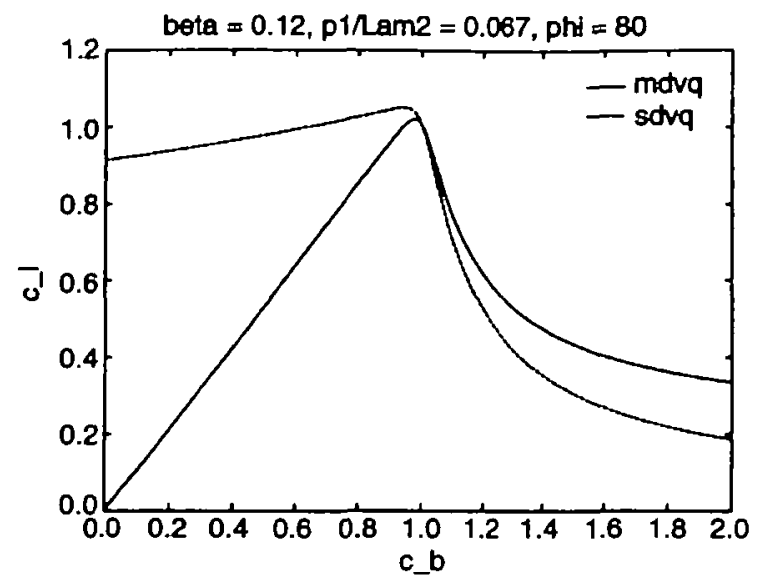

Fio. 1. The function $q_{1}\left(c_{b}\right)$ computed with $\beta=0.12, p_{1} / \Lambda_{2}=0.067$, and $\phi=80$, and $\alpha=0, \gamma=1-\beta+$ $p_{1} / \Lambda_{2}$. The curve labelled 'mdvq' (multiple delay, variable $Q$ ) is computed using $c_{t}=c_{b}$, while that labelled 'sdvq' (single delay, variable $Q$ ) has $c_{t}=1$.

where $C_{1}=C(\tau-1)$, and

$$
\varepsilon=\frac{1}{p_{6} p_{55}}, \quad q(C)=\frac{Q[f(C)]}{Q_{\mathrm{b}}[f(C)]} .
$$

Fowler et al (1993) did not account for variable delay, and in addition they took the bloodflow variables to be constant, $q=1$. We thus begin our analysis of the variable delay, variable cardiac output by analysing (3.11).

\subsection{One equation model results}

A typical form of $f(C)$ is as shown in Fig. 1. With a definition given by (3.5) and (2.17), we find (normally) that there is a unique equilibrium point $C^{*}$, say, and its linear stability is determined by the linearized equation

$$
\varepsilon \tilde{C}^{\prime}=q^{*}\left[f^{\prime *} \tilde{C}_{1}-\tilde{C}\right],
$$

where $q^{*}=q\left(C^{*}\right)$ and $f^{\prime *}=f^{\prime}\left(C^{*}\right)$. A Hopf bifurcation occurs if $f^{\prime *}<0$, at a value

$$
f^{\prime *}=\sec \omega,
$$

where $\omega$ is the first positive root $(\pi / 2<\omega<\pi)$ of

$$
\tan \omega=-\varepsilon \omega / q^{*},
$$

and the steady state is stable for $\left|f^{\prime *}\right|<\sec \omega$.

Increasing $\left|f^{\prime *}\right|$ is principally associated with increasing $\delta$, and a plot of $\varepsilon$ versus $\delta$ then gives a curve (see Fig. 2) below which the steady state is unstable. The effect of variable cardiac output is to alter $q^{*}$, and since $q=Q / Q_{\mathrm{b}}$ decreases with $c_{l}$ in the Grodins formulation (see (3.2)), this has the effect of pushing the stability curve down, i.e. variable 


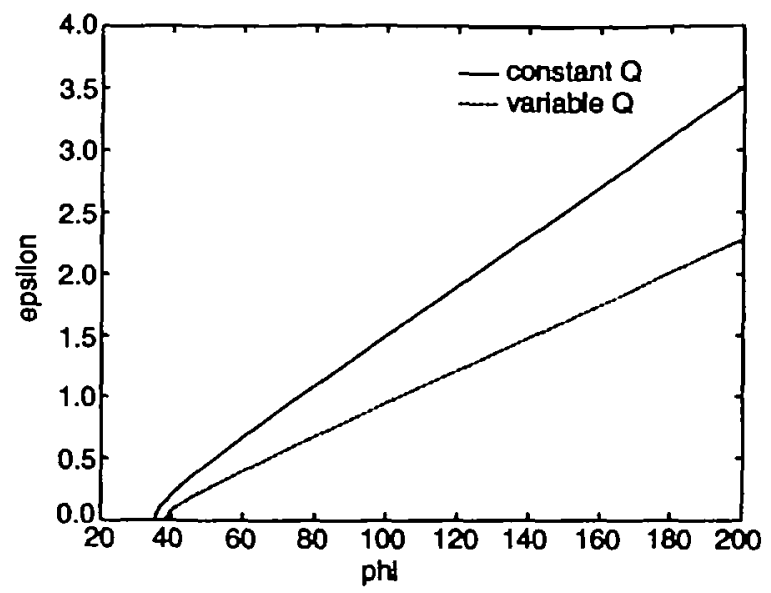

FIG. 2. Stability curves for variable and constant $Q$ with $\alpha=0, p_{1} / \Lambda_{2}=0.067$ and $\beta=0.12$. Instability occurs for values of $\varepsilon$ and $\phi$ below these curves.

cardiac output stabilizes the system because of the relatively increased brain blood flow. Of course, varying $q$ also shifts the value of $C^{*}$ and thus $f^{\prime *}$. Figure 2 indicates that the change to the stability curve is, or can be, substantial.

A question of some mathematical interest is the relationship between the dynamics of the differential-delay equation (3.11) and the map $C \mapsto f(C)$. It is clear from (3.14) and (3.15) that as $\varepsilon \rightarrow 0, f^{\prime *} \rightarrow-1$, which corresponds to the onset of instability in the map, and studies of this equation (with other forms of $f(C)$, for example the logistic term $f(C)=\lambda C(1-C)$ ) suggest that a period-doubling sequence of bifurcations of the map (when $\varepsilon=0$ ) are continued as curves in the $(\varepsilon, \delta)$ plane when $\varepsilon>0$. As $\varepsilon$ is reduced below the Hopf stability curve in Fig. 2, a periodic solution is created, and this represents the onset of periodic breathing. Figure 3 shows a typical form of this oscillation. Since the instability at $\varepsilon>0$ requires the instability of the map, we see from Fig. 1 that the steep negative slope of the single-delay curve is associated with instability, while the multipledelay curve (with $c_{\mathrm{t}}=c_{\mathrm{b}}$ in (2.17)) apparently precludes instability. In fact, the slow variation of $c_{\mathrm{t}}$ (if $p_{8} \ll p_{6}$ ) indicates that the single-delay curve will in fact be the more relevant for determining instability, and this is borne out in the numerical solutions.

The behaviour of the system as $\varepsilon$ is reduced further is associated with the behaviour of the map, as follows. With $\alpha=0$ and $\gamma=1-\beta,(2.17)$ is

$$
c_{1}=\frac{\beta Q_{\mathrm{b}} c_{\mathrm{b}}+\left(Q-\beta Q_{\mathrm{b}}\right) c_{\mathrm{t}}}{Q+\delta v\left(c_{\mathrm{b}}\right)} \text {. }
$$

With $Q, Q_{\mathrm{b}}=1, v \ll 1$ for $c_{\mathrm{b}}<1$, this is essentially a straight line of slope $\beta$,

$$
c_{1} \approx(1-\beta) c_{\mathrm{t}}+\beta c_{\mathrm{b}}, \quad c_{\mathrm{b}}<1,
$$

as is seen in Fig. 1. For $c_{\mathrm{b}}>1, v$ increases rapidly, and thus $c_{\text {l }}$ decreases. With $Q=Q_{\mathrm{b}}=$ 1 , we then have

$$
q_{1} \approx \frac{\beta c_{\mathrm{b}}+(1-\beta) c_{\mathrm{t}}}{1+\delta v}
$$




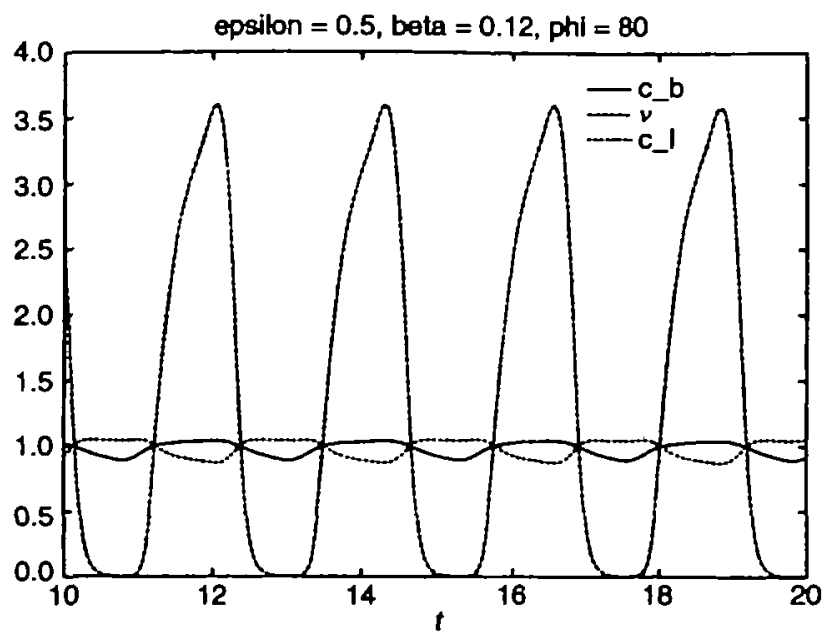

FIG. 3. Stable periodic oscillations at $\varepsilon=0.5, \beta=0.12, \phi=80, \alpha=0, p_{1} / \Lambda_{2}=0.067$ (and thus $\delta=5.36$ ). The single variable delay model is used, with $c_{t}=1$ in (2.17). The parameters $p_{6}$ and pss are taken as 2 and 1 respectively (hence the value of $\varepsilon$ ). The delay is thus $\sim$ pSS $\approx 1$, and notice that the oscillations have an approximate period of 2 pss.

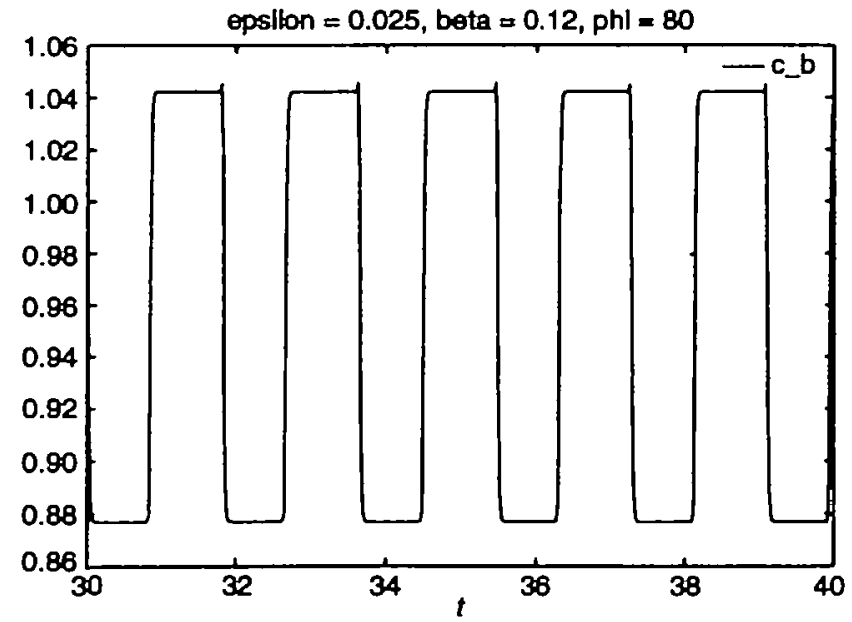

FIO. 4. Relaxation oscillations at $\varepsilon=0.025, \beta=0.12, \delta=5.36$.

another straight line with smaller slope (as in Fig. 1). Thus $f(C)$ has the typical form in Fig. 1, essentially a high value jumping to a low value. The steepness of the slope in the jump is associated with the value of $\delta$, increasing for increasing $\delta$.

This explains why increasing $\phi$ and thus $\delta$ is associated with instability, and also suggests the behaviour as $\varepsilon$ is decreased further. We have seen that the Hopf instability is the continuation to $\varepsilon>0$ of the period-doubling instability of the map when $\varepsilon=0$. If the map has a complete period-doubling sequence, then we can expect that each perioddoubling bifurcation will give rise to a nested sequence of curves in $\varepsilon>0$, so that as 


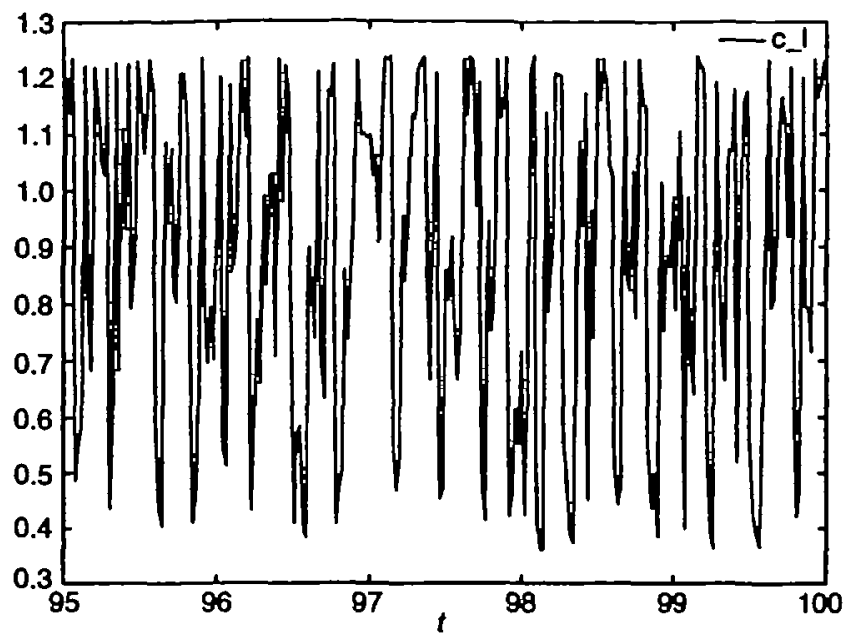

FIG. 5. Chaos in arterial $\mathrm{CO}_{2}$ concentration when $\delta=0.01, \beta=1.2, \gamma=0.2$ and $\delta=16$, for the single constant delay model.

$\varepsilon$ is decreased, a corresponding period-doubling sequence of bifurcations will occur, with chaotic solutions at small $\varepsilon$. Indeed, Fowler et al. (1993) found such behaviour when $\beta=1$, $\gamma=0.4, c_{t}=1$ (although with a different choice for $v(c)$ ), when the underlying map (3.16) resembles a unimodal map. However, at smaller (more realistic) values of $\beta$, the function $f(C)$ has the step shape indicated in Fig. 1, and it is clear that this map will not have a full period-doubling sequence; indeed a step function will only have the first period-doubling, with the period two orbit remaining stable thereafter. It is, therefore, not surprising that for low values of $\beta$, the periodic solution remains stable, becoming relaxational (Fig. 4) as $\varepsilon \rightarrow 0$. The absence of chaos in this case is consistent with discussion by an der Heiden \& Mackey (1982), and the relaxational form was studied by Chow \& Mallet-Paret (1985) and Chow et al. (1992).

It is of course also of mathematical interest to study the chaotic forms of the solution at higher $\beta$, and the relation of these to the dynamics of the underlying map. Figure 5 shows chaotic oscillations (for a single constant delay of PS5 $=1$ ) in arterial $\mathrm{CO}_{2}$ when $\beta=1.2, p_{1} / \Lambda_{2}=0.4$, so that $\gamma=0.2$ and also $\phi=40$, so that $\delta=16$. Farmer (1982) shows that the dimension of the attractor in these states is $O(1 / \varepsilon)$, consistent with the rapid oscillations over a time scale of $O(\varepsilon)$ which are evident. Numerical studies (Wattis, 1990) (see Fig. 6 for an example) also indicate that this kind of chaos arises from an initially constant concentration through a gradual erosion of the plateau by growing inter-plateau boundary layers. The map clearly exerts an influence on the differential delay equation as $\varepsilon \rightarrow 0$, but its precise nature remains opaque.

\subsection{Multiple delays, variable cardiac output}

The generalization we have made in the above results is to allow variable $Q$ and $Q_{\mathrm{b}}$ as functions of $c$; this leads to variable delays. A comment on the numerical method of 


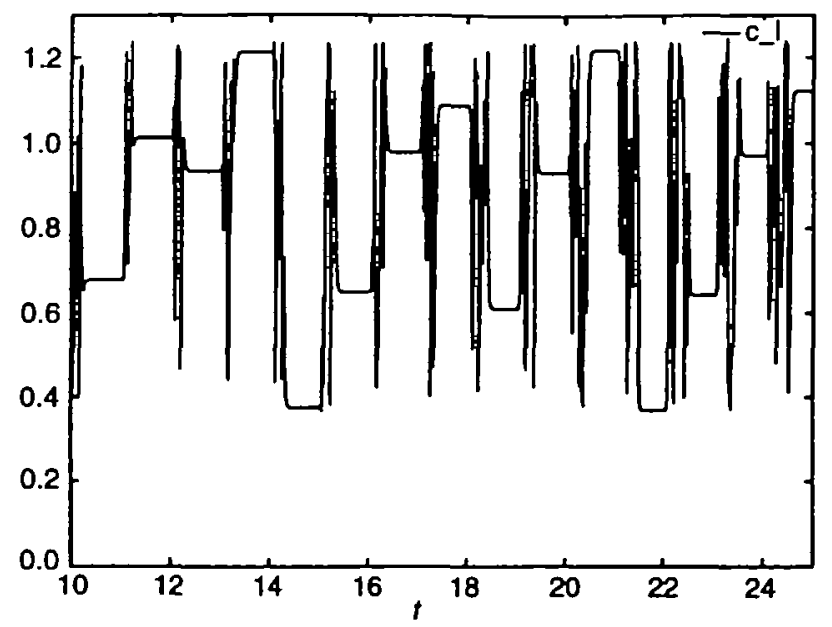

FIG. 6. The approach to the chaotic oscillations in Fig. 5 occurs through erosion of the plateans related by the underlying difference equation. (Note the different time scale compared with Fig. 5.)

solution is in order. For a variable delay $\tau$ determined by an equation such as

$$
p=\int_{t-\tau}^{t} Q[c(t)] \mathrm{d} t
$$

one option is to solve the differential equation

$$
\dot{\tau}=1-Q[c(t)] / Q[c(t-\tau)]
$$

this gives unstable results. Instead, we define

$$
J(t)=\int_{0}^{t} Q[c(t)] \mathrm{d} t
$$

so we solve

$$
\dot{J}=Q, J(0)=0,
$$

and simply choose $\tau$ (via linear interpolation between equal time steps) so that

$$
J(t)-J(t-\tau)=p
$$

(Linear interpolation to evaluate $c(t-\tau)$, etc., is also used in the case of constant delay, indeed the numerical results already presented use the variable delay code.) Baker and co-workers (Wille \& Baker, 1992, 1994) consider numerical methods for state-dependent delay diferential equations, the basic idea being that Runge-Kutta methods, which rely on smoothness of the equations for guaranteed accuracy, will work best in delay equations if the delayed arguments $t-\tau$ can be forced to lie on an appropriately chosen variable time stepped mesh. On the other hand, Press et al (1989) recommend the use of Runge-Kutta methods even for non-smooth equations, up to a point. Since the discontinuities in the 


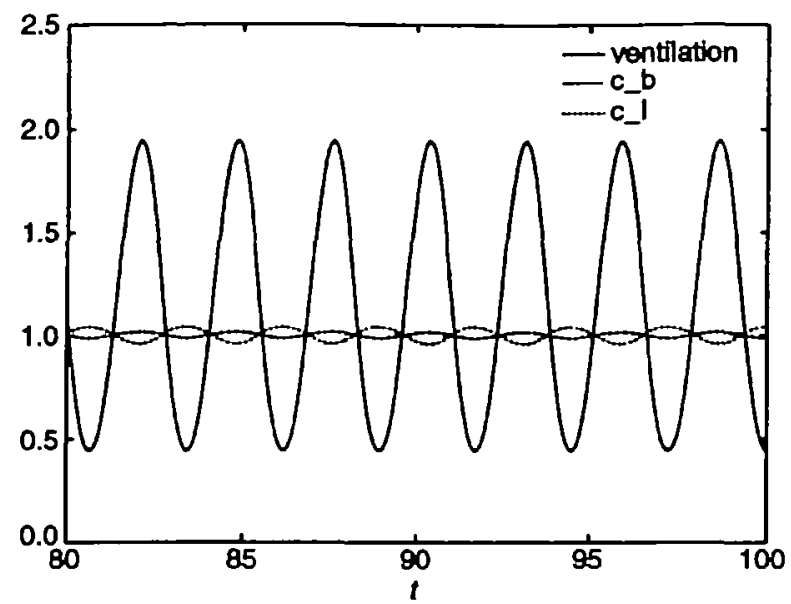

FIG. 7. Oscillations with multiple variable delays; the parameters are those of Fig. 3, except that $\epsilon=2, \phi=100$.

solution of a differential delay equation with smooth right-hand side (here, smooth $f(C)$ ) are shifted progressively to higher derivatives as $t$ increases, we can have some confidence that the Runge-Kutta method we use is satisfactory.

If we now further generalize to solve the two delay equations (2.5), together with variable $Q$ and $Q_{\mathrm{b}}$, and the two separate variable delays $\tau_{\mathrm{zb}}\left(=\tau_{\mathrm{at}}\right)$ and $\tau_{\mathrm{vt}}$, then we find that the presence of the second (venous) delay serves further to destabilize the system. Figure 7 shows a typical periodic oscillation when $\varepsilon=2$ and $\phi=100$, for the full multiple variable delay system, and this develops into a relaxational oscillation as $\varepsilon$ is reduced. It can be seen from Fig. 2 that these values are well in the stability range even for constant delays. On the other hand, this destabilization is due to the second delay in (2.17) rather than the second delay in (2.5); solving the system with the parameters of Fig. 7, but with $p_{8}=0.015$ rather than its default value of 0.15 , gives only a slightly different oscillatory response. This lack of effect of the second delay equation is a manifestation of the fact that at a value $p_{8} / p_{6}=0.22$, the $c_{1}$ equation really is slow. Figure 8 shows the effect of increasing $p_{8}$ to 0.5 , with other parameters being as in Fig. 3; evidently even this makes little practical difference.

\section{Discussion}

\subsection{Parameter interpretation}

The definitions of the parameters in the model are given by Fowler et al. (1993) and Kalamangalam (1995). The equations are given by (2.5), the delays by (2.11) and $c_{l}$ is given by (2.17). The independent parameters in these equations are $\alpha, \beta, \gamma, \delta, p_{56}, p_{26}$, $p_{55}, p_{8}, p_{6}$. The parameter $\alpha$ represents the inspired $\mathrm{CO}_{2}$, and is taken to be zero here.

The parameter $p_{55}$ is given by (Fowler et al., 1993)

$$
p_{55}=\frac{\alpha_{35}}{[Q][t]}
$$




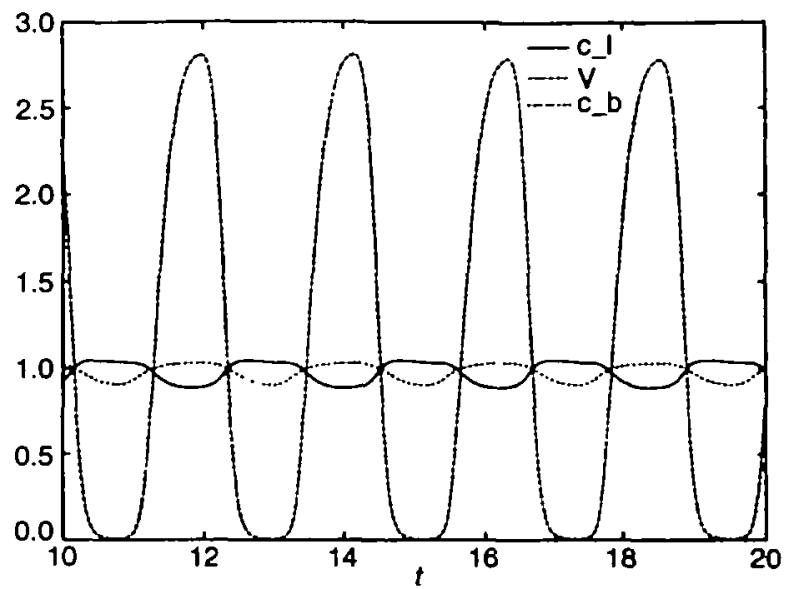

FIG. 8. The same parameter values are used as in figure 3, except that $p_{8}=0.5$.

The scale $[t]$ was chosen arbitrarily as $[t]=1 \mathrm{~min}$, and in fact it is natural to rescale $t \sim$ $p_{55}$ (corresponding to $\sim 10 \mathrm{~s}$ in normal subjects), and our subsequent discussion reflects this. Notice that if the typical Cheyne-Stokes cycle time of $1 \mathrm{~min}$ or more represents twice the lung-brain arterial delay, this would be associated with a three-fold reduction in cardiac output, such as might occur in patients with severe heart failure.

The parameter $p_{26}$ is given by

$$
p_{26}=\frac{\left[Q_{\mathrm{b}}\right]}{[Q]},
$$

and represents the ratio of the blood flow to the brain to the total cardiac output. If $Q$ is decreased, it is natural to expect a concomitant reduction in $Q_{b}$, but presumably not as much. The parameter $p_{56}$ in (2.11) is

$$
p_{56}=\frac{\alpha_{37}}{[Q][t]},
$$

but the clinically relevant parameter is the ratio (if $t \sim p_{55}$ )

$$
\frac{p_{56}}{p_{55}}=\frac{\alpha_{37}}{\alpha_{35}} \sim 2.7
$$

this represents the ratio of the venous/tissue delay $\tau_{v t}$ to the arterial/brain delay $\tau_{\mathbf{a b}}$, and appears dependent only on peripheral parameters.

Next, the rate parameters are $p_{6}$ and $p_{8}$. In terms of the time scale $p_{55}$, the clinically relevant parameters are

$$
\varepsilon=\frac{1}{p_{6} p_{55}}=\frac{[Q]}{\alpha_{35} k_{2}\left[Q_{b}\right]},
$$

where we take the $\mathrm{CO}_{2}$ scales to be equal, i.e. $\left[c_{\mathrm{ab}}\right]=\left[c_{b}\right]$; assuming also $\left[c_{\mathrm{at}}\right]=\left[c_{\mathrm{t}}\right]$, then the tissue $\mathrm{CO}_{2}$ rate parameter (relative to brain $\mathrm{CO}_{2}$ ) is

$$
\frac{p_{8}}{p_{6}}=\frac{k_{3}}{k_{2}} \frac{[Q]}{\left[Q_{b}\right]} \text {. }
$$


Finally the two dynamic parameters $\beta$ and $\delta$ are defined as follows:

$$
\beta=\frac{\Lambda_{1} p_{13}}{\Lambda_{2}}=p_{13}=\frac{\left[Q_{b}\right]}{[Q]}
$$

again assuming all $\mathrm{CO}_{2}$ scales are the same; the parameter $\delta$ is given by

$$
\delta=\frac{p_{1} \phi}{\Lambda_{2}}=\frac{\Delta \dot{V}}{\beta_{2}[Q]}
$$

where $\Delta \dot{V}=\phi[\dot{V}]$ is the change in ventilation that would result (extrapolatively) if the alveolar $\mathrm{CO}_{2}$ could be doubled from its normal value.

We see from these definitions that a critical parameter is $\beta$, which is in fact the ratio of brain blood flow to total cardiac output. Indeed, if blood flow alters, then it is this ratio which is of significance in determining the onset of periodic breathing.

According to this theory, as indicated in Fig. 2, periodic breathing will occur for sufficiently high $\delta$, which depends both on the sensitivity of the $\mathrm{CO}_{2}$ ventilation controller and on the cardiac output. In addition, $\varepsilon$ needs to be low, and this occurs if the fraction of blood flow to the brain is large. In particular, if $Q$ is reduced dramatically without a similar reduction in $Q_{b}$, then $\varepsilon$ is decreased: we thus identify reduced cardiac output as a primary cause of periodic breathing in this model. For example, a reduction of cardiac output by a third is sufficient to cause periodic breathing if the gain is $\phi=60, Q_{\mathrm{b}}$ is reduced by two-thirds, and the normal value of $\epsilon$ is 4 , rather than the 'normal' value of 8. Apart from cardiac output, this may depend on the value of $k_{2}^{-1}$, which is the brain compartment volume $\left(K_{\mathrm{B}}\right)$ in the Grodins model.

However, if $\varepsilon$ is reduced via a reduction in $[Q] /\left[Q_{\mathrm{b}}\right]$, then also $p_{26}$ decreases, $p_{8} / p_{6}$ decreases, $\beta$ increases. The decrease in $p_{8} / p_{6}$ implies that the single delay equation is more accurate, while the increase in $\beta$ promotes (via (3.16)) instability. Thus the effect of cardiac output variability on periodic breathing is due not to the absolute value of cardiac output $[Q]$, but to the ratio $[Q] /\left[Q_{\mathrm{b}}\right]$.

\section{Discussion}

A continuous ventilation model can be used to predict the onset of periodic breathing. We find that, for practical purposes, a single delay differential equation for brain $\mathrm{CO}_{2}$ concentration is sufficient to describe the principal features of the model. Two controlling parameters, $\varepsilon$ and $\delta$, exert control on the occurrence of periodic breathing, which is promoted at small $\varepsilon$ and large $\delta$. Large $\delta$ means a sensitive neurophysiological response to $\mathrm{CO}_{2}$ variations, while small $\varepsilon$ is associated with increased fraction of blood flow to the brain.

Typical estimates for these parameters in man are $\varepsilon=8, \delta=2$ (if $\phi=30$ ), while values $\varepsilon<0.4$ (for $\phi$ in the range 40-60, see Fig. 2) are then required for periodic breathing (larger for higher values of $\delta$ ), at least in the single delay model. It thus seems that periodic breathing in this model might represent a rather extreme occurrence, whereas in fact it is not uncommon. Two features of the model presented here may have relevance to this point. One is the assumption of a continuous ventilation model; our current opinion is that this will in fact be only a detail in the description of the phenomenon. The second 
is the description of the blood transport via a constant-velocity pipe flow. In reality, the branching of blood flow through arterioles and capillaries represents a modification of the physiological descriptions inherent in the model which may well cause less extreme fluctuations in the parameters to cause periodic breathing; this exploration awaits future study. It must also be pointed out, of course, as we discuss further below, that the inclusion of the venous delay has a significant destabilizing effect, although we have not been able to compute the stability limit explicitly in this case.

\subsection{Clinical relevance}

The two classical clinical conditions recognized to predispose to PB in adults are central nervous system disease, such as stroke (causing increase in the 'gain' of the central chemoreceptor) and left heart failure (causing increase in heart-to-brain blood transport delay) (Tobin \& Snyder, 1984; Yamashiro \& Kryger, 1993).

Note, however, that instability 'produced' by decreasing $[Q]$ is facilitated by a normal, or increased, $\left[Q_{\mathrm{b}}\right.$ ]: the widely accepted concept that heart failure leads to $\mathrm{PB}$ via increased delays is better thought of as being due to a combination of imbalance in the vascular ratio $Q / Q_{b}$ together with an increase of the effective gain $\delta$. Such a viewpoint suggests that increased $Q_{b}$, by itself can predispose to PB. We are aware that PB occurs in a wide variety of nervous system disorders: our proposal is that, at least in some of these pathologies (i.e. those associated with increased brain blood flow, such as inflammatory encephalopathies), cerebral hyperaemia may be a causative factor for $\mathrm{PB}$. In addition, the $Q / Q_{\mathrm{b}}$ ratio concept provides a possible mechanism for PB seen in vascular disorders, e.g. following surgery on major blood vessels (Reeder et ah, 1991): it is plausible that, following reconstructive aortic surgery, post-operative vessel spasm transiently decreases the $Q / Q_{\mathrm{b}}$ ratio, causing PB. We are not aware of any other mechanism proposed to explain PB in this situation, and our results, at the very least, are pointers to where to look further for answers.

Cycling of brain (cerebral) blood flow with PB has long been known, but only apparently recently has this been observed directly (Jenni et al, 1994; Franklin et al., 1997). Taken together with overnight polysomnographic recordings (i.e. simultaneous recordings of breathing movements, arterial blood gas saturations, and sleep state), this leads Franklin et al (1997) to view de novo fluctuations in brain function (as evidenced by sleep state and brain blood flow) as secondarily causing ventilatory (and consequently, blood gas) fluctuations. In effect, the chemical feedback hypothesis (which the majority of workers, including ourselves, have based work on), is considered inadequate for the causation of PB. Our demonstration of brain blood flow fluctuations coupled to PB shows that the chemical feedback hypothesis can very well accommodate the observation of cycling brain blood flow; in so far as our results go, this takes the debate of "brain state versus chemical feedback' in the causation of PB a step further in favour of the latter. To be sure, there are finer points not addressed by us: brain blood flow was seen to oscillate in phase with breathing in the observations of Franklin et al. (1997), whereas if $Q=Q\left(c_{1}\right)$ as we have assumed here, then we obtain an out-of-phase relation. Further observations and modelling are needed to clarify this issue (for instance, the report of Jenni et al. (1994) contradicts Franklin et al. (1997) by observing in-phase cerebral blood flow oscillations). In addition, more specific modelling of the way in which blood gases affect cardiac output needs to be done. 


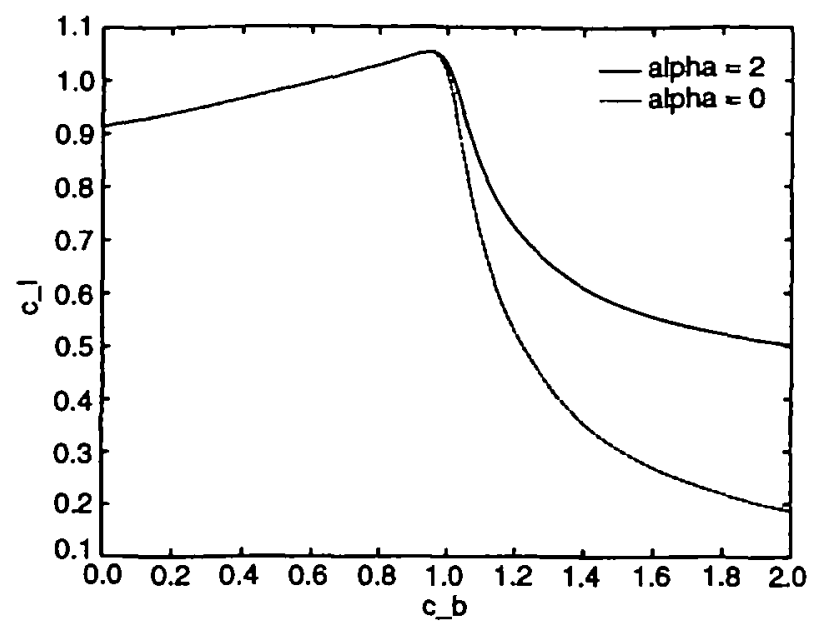

FIG. 9. A comparison of the forms of the function $q\left(c_{b}\right)$ when $\alpha=0$ and $\alpha=2$.

Periodic breathing may be reduced, or abolished, in clinical situations by increasing the concentration of oxygen or carbon dioxide in inspired air (Tobin \& Snyder, 1984; Steens et al., 1994; Franklin et al., 1997). The effect of increasing inspired $\mathrm{CO}_{2}$ is easily verified in our model. Recall that the tendency of the model to oscillate depends on the slope of the map given by (2.17), or in Fig. 1, at its fixed point, in addition to the size of $\epsilon$; parametric variations that reduce this slope have a stabilising effect. The fraction of inspired $\mathrm{CO}_{2}$ is given by the constant $\mu_{1}$ in our model (see Fowler et al., 1993) and appears in the definition of the dimensionless parameter $\alpha$, which can be written in the form

$$
\alpha=\frac{\delta \mu_{1}}{\left[c_{1}\right]} \text {. }
$$

Increasing $\alpha$ from its usual value of zero makes the slope of the decreasing portion of the map less negative (see Fig. 9), consequently stabilizing oscillatory tendencies in the model. Figure 10 shows the stability curves computed with the same parameters as in Fig. 2, but with the two separate values of $\mu_{1} /\left[c_{1}\right]=0$ and $\mu_{1} /\left[c_{1}\right]=0.5$. The model is significantly stabilized. We cannot comment on the influence of increasing inspired oxygen because ours has been an exclusively $\mathrm{CO}_{2}$-based study, but it is pertinent here to clarify the overall scope of our results and comment on the observations of others. Respiration, we know, is dependent on both the hypoxic (mediated by the peripheral receptor) and the hypercapnoeic (largely mediated by the central receptor) 'drive'; our study has focussed on cardiorespiratory dynamics mediated by the $\mathrm{CO}_{2}$-sensitive central receptor, which is an adequate description of the whole system, providing there is no significant hypoxia (i.e. hypoxia sufficient to recruit the response of the peripheral receptor). In this situation, oxygen no longer determines respiration, but is certainly affected by it-cyclical variations in breathing (i.e. PB) will cause arterial $\mathrm{O}_{2}$ to fluctuate, but we are assuming that these 'desaturations' (drops in oxygen partial pressures) are mild and do not provoke a significant response from the peripheral receptor. On the other hand, there are situations of clinically significant hypoxia (namely ascent to high altitude, or lung disease) that accompany PB, 


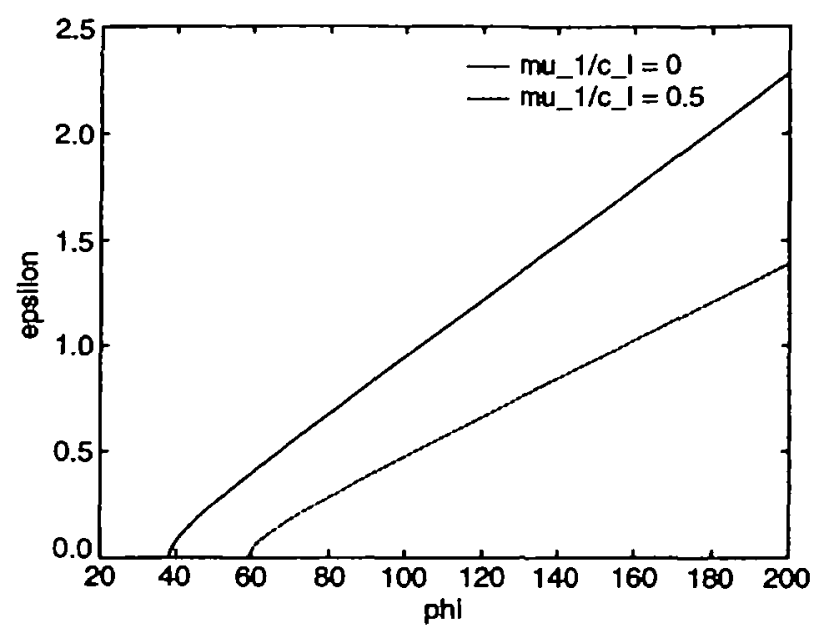

FIG. 10. Stability maps for $\mu_{1} /\left[q_{1}\right]=0$ and $\mu_{1} /\left[c_{1}\right]=0.5$, other parameters as for Fig. 2 .

and these are clearly instances where the hypoxic drive must be taken into account. Peripheral receptor mediated PB has a faster cycle time $(\approx 20 \mathrm{~s}$; see West et al. (1986), for instance) than central receptor mediated PB (which may have a period of up to a few minutes). It may be difficult in a given clinical situation to disentangle the relative contributions of peripheral and central receptors in a patient breathing periodically, but conceptually dissociating them is helpful in interpreting experimental results. The article of Steens et al. (1994) reports on the occurrence of PB in patients with severe left heart failure; the typical cycle length of $\mathrm{PB}$ in these patients was about $60 \mathrm{~s}$, and PB was consistently shown to disappear on increasing the concentration of inspired $\mathrm{CO}_{2}$. The time period of $\mathrm{PB}$ observed by these workers, along with the effect of increasing inspired $\mathrm{CO}_{2}$, point to an exclusive central receptor mediated dynamics in these patients. On the other hand, the report of Franklin et al. (1997) documents PB in a mixed group of patients (a few with heart failure, a few with a previous stroke, and a few with both). Cycle lengths are reported as averaging about $52 \mathrm{~s}$. On increasing the concentration of inspired oxygen, the following were observed: oscillations in arterial $\mathrm{O}_{2}$ were diminished, $\mathrm{PB}$ cycle length was increased, and the cycling of cardiovascular (blood pressure and cerebral blood velocity) variables was unchanged. Franklin et aL (1997) interpreted these findings as evidence that blood gas changes were not sufficient to account for $\mathrm{PB}$ (because the cycling of $\mathrm{O}_{2}$ had been reduced), and put forward their case for PB being primarily caused by fluctuating cerebral function (as detailed above). We have a different explanation, assuming that PB in these patients had contributions from both the central and peripheral receptors. The cycling time would have had a relatively fast component from the peripheral receptor, and a slower one from the central. Administration of oxygen would suppress PB arising from the peripheral receptor, leaving the slower frequency to increase the length of the observed cycle. Fluctuations in arterial $\mathrm{O}_{2}$ would naturally be smoothed over by the increased inspired fraction, but that would have no effect on the $\mathrm{CO}_{2}$-driven dynamics of the central receptor. Periodic breathing would thus continue (perhaps less vigorously), accompanied by the observed cardiovascular changes. 


\section{Conclusions}

For mathematicians, perhaps the best known simple model of periodic breathing is that due to Mackey \& Glass (1977); it balances the generation of $\mathrm{CO}_{2}$ by the tissues with its evacuation by gas exchange with the lungs. In this paper and its predecessor (Fowler $e t$ $a L, 1993)$, we have shown that, while it is indeed the case that a single delay differential equation can represent the chemical control of the respiratory system, the dynamics of the system are determined by the interaction between the $\mathrm{CO}_{2}$ in the different compartments of the body, and production is essentially irrelevant.

We have extended our previous results in two ways. First, by interpreting the meaning of the principal controlling parameters, we have confirmed that the model respiratory system is subject to oscillatory instability when the cardiac output is reduced, as in patients with heart failure. Furthermore, the cycle time is then approximately twice the lung to brain arterial delay time, which is consistent with reduction of cardiac output by a factor of three or more, when compared with observations (Vandenaardweg \& Karemaker, 1991).

Secondly, we have shown that the inclusion of variable cardiac response to blood gas concentrations, which leads to variable delays, has a stabilising effect, in contrast to the separate inclusion of venous delay from tissues to lung; on the other hand, the tissue $\mathrm{CO}_{2}$ level is slowly varying, and appears not to affect the dynamics significantly.

We are not aware that the importance of the venous delay has been appreciated in the literature hitherto. The experiment of Guyton et al. (1956) consisted of artificially increasing arterial delay in an anaesthetized animal-they observed periodic breathing, but only at a 10-fold increase in delay. Our results suggest that introducing a (smaller) delay each at the arterial and venous sites would have been a better strategy (sluggish circulation would certainly increase both the arterial and venous delays in the intact animal or human, and this is represented in our model by the constancy of the ratio $p_{56} / p_{55}-$ see equation (4.4)). We also know (see Goldberger et al., 1984, for instance) that a failing heart shows less 'variability' (i.e. spontaneous fluctuations in heart rate) than a healthy one: this reflects its inability to respond in the normal healthy way to (constantly changing) environmental influences. Such inability would presumably include a poor response to varying blood gas concentrations, effectively decoupling the cardiac outputbrain blood flow variables from the rest of the model; the result is constant delays, and as we have just described, a greater propensity to oscillatory instability.

In addition to the confirmation of others' quantitative demonstration of heart failureand brain pathology-induced PB, we have described several novelties: (i) a further mechanism underlying $\mathrm{PB}$ in heart failure, (ii) a possible mechanism for $\mathrm{PB}$ in vascular disorders, (iii) the introduction of the concept of venous delays playing a role in the genesis of $\mathrm{PB}$, (iv) re-interpretation of experimental observations of PB-synchronous cerebral blood flow oscillations; ( $v$ ) confirmation of the effect of increasing inspired $\mathrm{CO}_{2}$ concentration on $\mathrm{PB}$, which led to an alternative explanation of the effect of increasing inspired $\mathrm{O}_{2}$ in clinical PB.

Periodic breathing, as is well-known, is not restricted to pathologies of the heart and brain. It is a regular feature in humans taken to high altitude, in normal and preterm infants, in congestive disorders of the lung in adults and even during normal sleep. In all of these conditions, however, there is a degree of hypoxia which contributes to, or is responsible for it. Taking the effects of hypoxia into account (via dynamics at the peripheral receptor) would be the next step in the furtherance of the present study. 


\section{Acknowledgements}

Much of this research was supported by a Wellcome Prize Studentship to GPK.

\section{REFERENCES}

AN DER HEIDEN, U. \& MACKEY, M. C. 1982 The dynamics of production and destruction: analytic insight into complex behaviour. J. Math BioL 16, 75-101.

CARLEY, D. W. \& SHANNON, D. C. 1988 A minimal mathematical model of human periodic breathing. J. Appl. Physiol 65, 1400-1409.

ChOW, S. N. \& MALLET-PARET, J. 1985 Singularly perturbed delay-differential equations. Coupled Nonlinear Oscillators (J. Chandra \& A. C. Scott eds). North Holland, Amsterdam: pp. 7-12.

Chow, S. N., Hale, J. K., \& HUANG, W. 1992 From sine waves to square waves in delay equations. Proc. R. Soc. Edinb. 120A, 223-229.

ElHefnawy, A., Saidel, G. M., BruCe, E. N., \& Cherniak, N. S. 1990 Stability analysis of the $\mathrm{CO}_{2}$ control of ventilation. J. Appl Physiol 69, 498-503.

FARMER, J. D. 1982 Chaotic attractors of an infinite-dimensional dynamical system. Physica D4, 366-393,

Fowler, A. C., Kalamangalam, G. P., \& Kember, G. C. 1993 A mathematical analysis of the Grodins model of respiratory control. IMA J. Maths. Appl. Med. Biol. 10, 249-280.

FrankLIN, K. A., SANDSTrom, E., Johansson, G., \& Balfors, E. M. 1997 Hemodynamics, cerebral circulation, and oxygen saturation in Cheyne-Stokes respiration. J. Appl. Physiol 83, 1184-1191.

Goldberger, A. L., FindLey, L. J., BlackBURN, M. R., \& MANDELL, A. J. 1984 Nonlinear dynamics in heart failure: implications of long-wavelength cardiopulmonary oscillations. Amer. Hear J. 107, 612-615.

Grodins, F. S., BUELL, J., \& BART, A. J. 1967 Mathematical analysis and digital simulation of the respiratory control system. J. AppL. PhysioL 22, 260-276.

GuYToN, A. C., CRowell, J. W., \& MOORE, J. W. 1956 Basic oscillating mechanism of CheyneStokes breathing. Amer. J. Physiol. 187, 395-398.

JenNi, O. G., BuCher, H. U., VON Siebenthal, K., WOLF, M., KeEl, M., \& Duc, G. 1994 Cyclical variations in cerebral blood volume during periodic breathing. Acta Paediatrica 83, 1095-1096.

Kalamangalam, G. P. 1995 Nonlinear oscillations and chaos in chemical cardiorespiratory control. D. Phil Thesis, University of Oxford.

KAPLAN, D. T. \& TALAJIC, M. 1991 Dynamics of heart rate. Chaos 1, 251-256.

KhOO, M. C. K., Kronauer, R. E., STROHL, K. P., \& Slutsky, A. S. 1982 Factors inducing periodic breathing in humans: a general model. J. Appl Physiol. 53, 644-659.

KITNEY, R. I. \& ROMPELMAN, O. 1987 The beat-by-beat investigation of cardiovascular function. Oxford University Press.

LONGOBARDO, G. S., GOTHE, B., \& ChERNIAK, N. S. 1989 Factors affecting respiratory system stability. Ann. Biomed. Engng. 17, 377-396.

MACKEY, M. C. \& GLASS, L. 1977 Oscillations and chaos in physiological control systems. Science 197, 287-289.

Mortara, A., Sleight, P., Pinna, G. D., Maestri, R., PrPa, A., LaRovere, M. T., COBELLI, F., \& TAVAZZI, L. 1997 Abnormal awake respiratory patterns are common in chronic heart failure and may prevent evaluation of autonomic tone by measures of heartrate variability. Circulation 96, 246-252. 
PETERSEn, E. S. \& CUNNINGHaM, D. J. C. 1988 Respiratory physiology. The respiratory system (D. G. Cramp \& E. R. Carson eds). Croom Helm, London: pp. 13-96.

Press, W. H., Flannery, B. P., Teukolsky, S. A., \& Vetterling, W. T. 1989 Numerical recipes. Cambridge: C.U.P..

Quaranta, A. J., D'Alonzo, G. E., \& KRAChmaN, S. L. 1997 Cheyne-Stokes respiration during sleep in congestive heart failure. Chest 111, 467-473.

Reeder, M. K., Goldman, M. D., Loh, L., Muir, A. D., \& Casey, K. R. 1991 Haemodynamic effects of periodic ventilation: abolition with supplementary oxygen. $B r i t . J$. Anaesth 67, 326-328.

SMITH, H. L. 1993 Reduction of structured population models to threshold-type delay equations and functional differential equations: a case-study. Math. Biosci. 113, 1-23.

Steens, R. D., Millar, T. W., Xiaoling, S., Biberdorf, D., BuCKle, P., Ahmed, M., \& KRYGER, M. H. 1994 Effect of inhaled $3 \% \mathrm{CO}_{2}$ on Cheyne-Stokes respiration in congestive heart failure. Sleep 17, 61-68.

TOBIN, M. J. \& SNYDER, J. V. 1984 Cheyne-Stokes respiration revisited: controversies and implications. Crit. Care Med 12, 882-887.

VANDENAARDWEG, J. G. \& KAREMAKER, J. M. 1991 Respiratory variability and associated cardiovascular changes in adults at rest. Clin. Physiol 11, 95-118.

WATTIS, J. A. D. 1990 Bifurcations and chaos in a differential-delay equation. M. Sc. Dissertation, Oxford University.

West, J. B., Peters, R. M., Aksnes, G. K. H., Milledge, J. S., \& Schoene, R. B. 1986 Nocturnal periodic breathing at 6300 and $8050 \mathrm{~m}$. J. AppL Physiol 61, 280-287.

WILlE, D. R. \& BAKER, C. T. H. 1992 The tracking of derivative discontinuities in systems of delay-differential equations. AppL Numer. Math. 9, $209-222$.

WILlE, D. R. \& BAKER, C. T. H. 1994 Stepsize control and continuity consistency for statedependent delay-differential equations. J. Comput. AppL Math. 53, 163-170.

YAMASHIRO, Y. \& KRYGER, M. H. 1993 Review: sleep in heart failure. Sleep 16, 513-523. 
